\title{
Left atrial appendage aneurysm presenting with chronic cough
}

\author{
M. Toufan ${ }^{1}$ L. Pourafkari ${ }^{1,2}$ • A. Afrasiabi ${ }^{1}$ M. Sohrabi ${ }^{1}$ N. D. Nader ${ }^{2}$
}

(C) The Author(s) 2017. This article is an open access publication.

A 32-year-old female presented with a 6-month history of worsening non-productive chronic cough and palpitations on moderate exercise. Chest X-ray showed an increased cardiothoracic ratio with a prominent left heart border. She underwent transthoracic echocardiography in which a large cystic structure with compressive effect on the left ventricle was identified (Fig. 1a). Further evaluation by transoesophageal echocardiography proved the structure to be a large left atrial appendage aneurysm (LAAA) $(7.7 \times$ $4.4 \mathrm{~cm}$ ) with a $1 \mathrm{~cm}$ entrance to the atrial cavity (Fig. 1b). The patient underwent aneurysm resection under cardiopulmonary bypass. Fig. 1c shows the resected aneurysm, which had a very thin wall. The postoperative course was uneventful.
LAAA are rarely encountered and generally present with palpitations, chest pain, dyspnoea or thromboembolic events [1]. The chronic cough could have been caused by the mechanical airway compression by LAAA. Once diagnosed, surgery is warranted regardless of presence of symptoms.

Conflict of interest M. Toufan, L. Pourafkari, A. Afrasiabi, M. Sohrabi and N.D. Nader declare that they have no competing interests.

Open Access This article is distributed under the terms of the Creative Commons Attribution 4.0 International License (http:// creativecommons.org/licenses/by/4.0/), which permits unrestricted use, distribution, and reproduction in any medium, provided you give appropriate credit to the original author(s) and the source, provide a link to the Creative Commons license, and indicate if changes were made.

\section{References}

1. Aryal MR, Hakim FA, Ghimire S, et al. Left atrial appendage aneurysm: a systematic review of 82 cases. Echocardiography. 2014;31:1312-8.
Electronic supplementary material The online version of this article (doi: 10.1007/s12471-017-1021-0) contains supplementary material, which is available to authorized users.

N. D. Nader

nadernd@gmail.com

1 Cardiovascular Research Center, Tabriz University of Medical Sciences, Tabriz, Iran

2 Department of Anesthesiology, University at Buffalo, New York, USA 

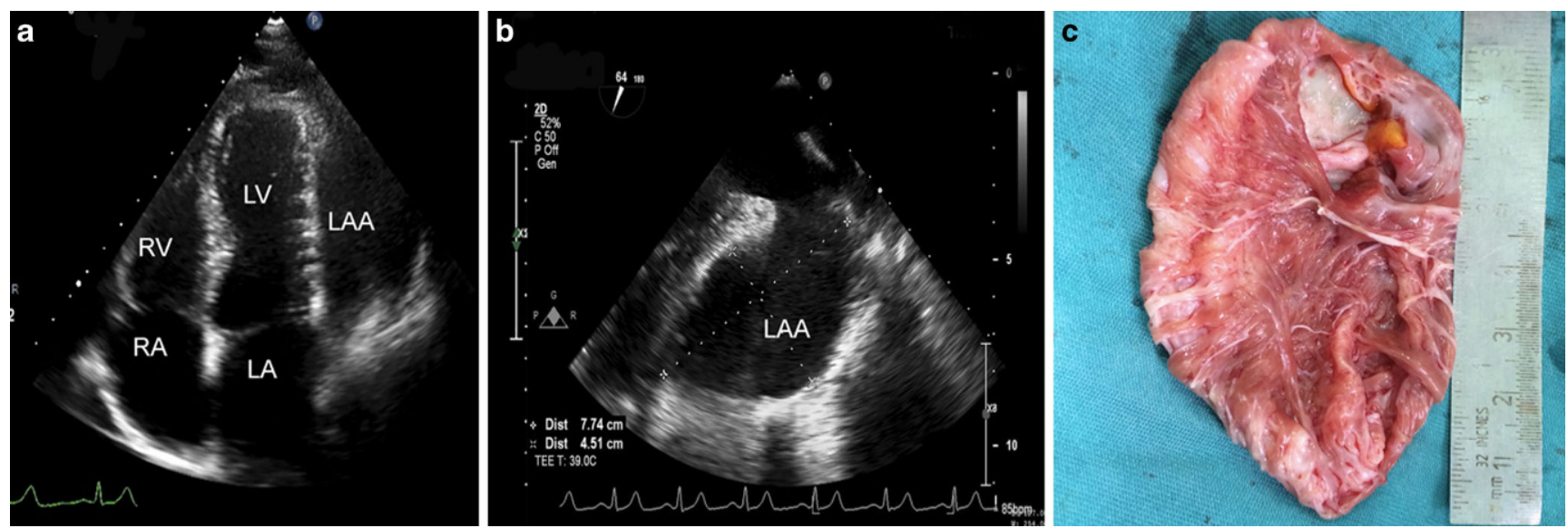

Fig. 1 a Transthoracic four chamber echocardiogram showing the aneurysmal left atrial appendage with compressive effect on left ventricle, b Transoesophageal echocardiogram obtained at mid-oesophageal level in $60^{\circ}$ showing the left atrial appendage aneurysm, c Intraoperative image showing the resected left atrial appendage aneurysm, $L A$ left atrium, $L A A$ left atrial appendage, $L V$ left ventricle, $R A$ right atrium, $R V$ right ventricle 